\title{
SISTEMA DE VALORES EN ESTUDIANTES DE QUINTO AÑO DE SECUNDARIA DE LIMA METROPOLITANA PERTENECIENTES A DIFERENTES ESTRATOS SOCIOECONÓMICOS ${ }^{1}$
}

Miguel Escurra Mayaute ${ }^{1}$

Se estudió la estructura del sistema de valores de los estudiantes de quinto año de secundaria de Lima Metropolitana, pertenecientes a diferentes estratos socioeconómicos, utilizando como marco conceptual de la Teoría de Schwartz. Los participantes correspondieron a una muestra probabilística estratificada de 1197 alumnos pertenecientes de manera representativa a las diferentes USE de Lima Metropolitana. Los hallazgos del análisis psicométrico demostraron la validez de contenido, la validez de constructo y la confiabilidad del Cuestionario de Valores de Schwartz (PVQ). Los resultados alcanzados indicaron que los puntajes de los valores del PVQ en los alumnos de quinto año de Lima Metropolitana presentaron una adecuada aproximación a la distribución normal. El análisis comparativo por estrato socioeconómico indicó que tradición y seguridad predominan en los alumnos del estrato socioeconórnico bajo. El poder predomina en el estrato medio. La benevolencia predomina en el estrato alto. La autodirección, el hedonismo y el logro predominan tanto en los estratos alto y medio. Mientras que la comparación por sexo permitió observar que la benevolencia, universalismo y la seguridad predominan en las mujeres y los valores de logro y poder predominan en los varones. Finalmente se encontró que en la muestra estudiada existen tres tipos de grupos según la forma de responder a los valores.

PALABRAS CLAVE: Valores, análisis psicométrico, confiabilidad, validez, estrato socioeconómico.

It was studied the structure of the values system of senior high schools students belonging to different socio-economic levels using Schwartz Theory as a framework. The participants were 1197 students sampled on a stratified probabilistic way, belonging to all school districts (USES) of Lima, Peru. The results of the psychometric analysis allowed to obtain the validity content index, the construct validity and the reliability index of the Schwartz Values Questionnaire (PVQ). The results showed that the scores of the students of the PVQ reached a normal distribution. The comparative analysis of the socioeconomic levels indicated that values of tradition and security prevail in low socioeconomic students. The value of power prevails in students of middle socioeconomic level and the benevolence value prevails in the high socio-economic level. The values of self direccion, hedonism and achievement prevail at the high and middle social economic levels. The comparison for sex allowed to observe that values as benevolence, universalism and security prevail in women and values of achievement and power prevail in males. Finally it was found three kind of profiles acoord to the form of responding to the PVQ.

KEY WORDS: Value, psychometric analysis, reliability, validity, socio-econornic levels.

\footnotetext{
${ }^{1}$ Participaron en la investigación: Ana Delgado Vásquez, Rosario Quesada Murillo, Amparo Sotil Brioso, Juan Pequeña Constantino, Gilmar Guevara Ortega, Roger Elizalde Bravo, Gerardo Rivas Castro, Rolando Solis Nano, Julio Santos Islas y Tomy Flores Lezama. Comunicaciones a: mescuITa@peru.com
} 


\section{INTRODUCCION}

En la actualidad constantemente se habla de una crisis profunda en los valores de nuestra sociedad, pero sin embargo, no se han llevado a cabo mayores estudios que de forma sistemática presenten los perfiles valorativos de los diferentes grupos sociales que la conforman. Cotidianamente nos enfrentamos a situaciones en las que debemos elegir entre metas contradictorias o vías contradictorias para conseguirlas. Para decidir en esas situaciones conflictivas, las personas se basan en preferencias relativamente abstractas y estables, es decir sus valores.

Los valores son concepciones de lo deseable, que influyen en las formas como la gente evalúa eventos y elige vías de acción (Kluckhohn, 1951). Es en éste sentido que los valores sirven de base tanto para las actitudes como para las conductas. La investigación psicológica y social han prestado especial atención a los valores personales como criterios para juzgar la realidad social y para orientar las actitudes y las conductas de individuos y grupos (Williams, 1979).

Una parte importante de esa investigación surge del trabajo clásico de Rokeach (1973), que desarrolló un instrumento para la medición de la jerarquía de valores personales y un procedimiento simple para influir sobre las actitudes y las conductas individuales mediante el suministro de información que llevará a la persona a afrontar críticamente sus propios valores. El progreso más importante en este campo ha sido el reciente desarrollo por Schwartz de una teoría integral sobre la estructura del sistema de valores que ha permitido sistematizar el estudio de los valores y el de su relación con las conductas (Schwartz \& Bilsky, 1987, 1990; en Schwartz, 1992, 1994, 2000).

Schwarlz (1996) propone utilizar un conjunto comprensivo de tipos de valores motivacionales probados transculturalmente. La comprensividad del sistema de tipos hace improbable que tipos relevantes de valores se consideren de forma aislada o incluso sean omitidos en el estudio de su relación con conductas, como podía suceder en los estudios clásicos de valores (Rokeach, 1973). Cada uno de esos tipos de valores es representado por varios valores combinados para formar índices fiables de prioridades valorativas. Finalmente, la teoría propuesta conceptualiza el conjunto de tipos motivacionales en un sistema integrado, que permite relacionar de forma organizada y coherente las prioridades de valores a otras variables.

\section{Teoría de los valores de Schwartz}

Schwartz, ha desarrollado un amplio proyecto de investigación para evaluar diferencias interculturales con respecto a una serie de tipos motivacionales, en los que agrupa a cincuenta y ocho valores. El proyecto ha incluido alrededor de sesenta países y a partir de los datos analizados es posible afirmar que existe una estructura universal en torno a algunas dimensiones básicas valorativas y que existen también diferencias entre las culturas estudiadas, asumiendo a los países y a ciertas regiones como Europa o América Latina como culturas (Schwartz y Sagie 2000; Schwartz, Sagiv \& Boehnke, 2000).

Esto sugiere que existen diversos modos por los que diferentes grupos sociales organizan la comprensión del mundo. Schwartz, construyó su mapa conceptual acerca de los valores subrayando que estos eran:

a) Conceptos o creencias.

b) Pertenecen a estados finales de ser o son conductas deseables.

c) Trascienden a situaciones específicas.

d) Guían la selección o la evaluación de la conducta.

e) Están ordenados por su importancia relativa para un individuo o una cultura. 
La identificación de aspectos universales del contenido de los valores y de su estructura hace posible investigar aspectos culturales específicos. La estructura se refiere a las relaciones de conflicto y compatibilidad entre los valores, encontradas en todas las culturas estudiadas, no a su importancia relativa para un grupo o un individuo. A esto último se refieren en la terminología de Schwartz las prioridades valorativas o las jerarquías de valores.

El modelo estructural de valores de Schwartz (Schwartz y Bilsky, 1987, 1990; Schwartz, 1992, 2000), postula que el principal aspecto de contenido que organiza el sistema de valores es el tipo de meta motivacional que representa. El modelo recibió un primer apoyo empírico en un estudio de siete países utilizando la Escala de Valores de Rokeach (RVS) (Schwartz y Bilsky, 1990). Schwartz (1992) presenta una verificación más amplia del modelo en 40 muestras de 20 países, aplicando un nuevo cuestionario de valores (Schwartz Value Survey - SVS) que intenta solucionar las limitaciones del RVS en la representación de todos los tipos básicos de valores.

Los tipos básicos de valores han recibido apoyo empírico en la interpretación de la organización del sistema de valores en 97 muestras de 44 países de todos los continentes habitados, con lo que pueden considerarse casi universales (Schwartz, 1985, 1996,2000; Schwartz \& Sagiv, 1995). Los resultados empíricos obtenidos evidencian también coherencia con las variables consideradas para analizar la validez ecológica del modelo.

Los tipos motivacionales de valores de acuerdo a sus metas según el modelo teórico de Schwartz (1996), son los siguientes:

1. Hedonismo: implica la búsqueda de obtener placer y gratificación sensual para la persona.

2. Seguridad: alude a conseguir seguridad, armonía y estabilidad en la sociedad, en las relaciones interpersonales y en la persona, incluye aspectos asociados a la seguridad familiar, la seguridad nacional, el orden social, la limpieza, la reciprocidad de favores, el sentimiento de pertenencia y el tener buena salud.

3. Poder: se relaciona con obtener posición y prestigio social, controlo dominio sobre personas y recursos, lograr poder social, autoridad, riqueza, conservar la imagen pública y alcanzar reconocimiento social.

4. Logro: se refiere a obtener el éxito personal como resultado de la demostración de competencia según las normas sociales, más que de acuerdo a criterios internos de excelencia que estarían mejor reflejados en valores de Autodirección. Tanto los valores de Poder como los de Logro se relacionan a la estima social, pero mientras aquellos se refieren al sistema social más general, éstos se aplican a las interacciones sociales concretas como son lograr éxito, ser capaz, ser ambicioso, ser influyente e inteligente.

5. Autodirección: alude a lograr la independencia en el pensamiento, la toma de decisiones y la acción, creación y exploración como son: la creatividad, libertad, elegir sus propias metas, ser curioso e independiente.

6. Conformidad: implica limitar las acciones, inclinaciones e impulsos que podrían trastornar o dañar a otros y violar expectativas o normas sociales, generalmente en las interacciones cotidianas con personas cercanas como es ser: obediente, auto disciplinado y tener buenos modales.

7. Universalismo: alude a la comprensión, aprecio, tolerancia y atención hacia el bienestar de toda la gente y hacia la naturaleza. Este tipo integra valores relacionados con la madurez como es la tolerancia, sabiduría, un mundo de belleza, protección del medio ambiente y con el bienestar social en general como en la justicia social, igualdad y lograr un mundo en paz. 
8. Estimulación: se refiere a tener estimulación, novedad y retos en la vida como es el caso de tener una vida variada, una vida excitante y ser osado o atrevido.

9. Tradición;.. implica respetar, comprometerse y aceptar las costumbres e ideas que la cultura tradicional o la religión imponen a la persona como es el respeto por la tradición, ser devoto, humilde y moderado.

10. Benevolencia: alude a preservar y reforzar el bienestar de las personas cercanas con quien uno está en contacto personal frecuente. Mientras los valores prosociales universalistas se aplican sin distinción a todas las personas, los de Benevolencia expresan una relación de preferencia hacia quien es considerado perteneciente a un mismo grupo social o endogrupo frente al exogrupo. Como es el caso de ayudar, honestidad, perdonar, lealtad y responsabilidad.

El modelo también define relaciones dinámicas entre los tipos de valores. Las limitaciones de carácter lógico y práctico para dar prioridad simultánea a diferentes valores (y las consecuencias psicológicas y sociales de las acciones para conseguirlos) determinan relaciones de contradicción o compatibilidad en el logro de los distintos tipos de valores. Por ejemplo, las acciones que intenten expresar obediencia (Conformidad) serán compatibles lógica y prácticamente con acciones para respetar la tradición (Tradición) pero seguramente serán contradictorias con acciones para obtener independencia personal (Autodirección). Como resultado de estas relaciones dinámicas, los diez tipos de valores se pueden agrupar en cuatro tipos de orden superior estructurados en dos dimensiones bipolares (Schwartz, 1992):

a. Trascendencia (Benevolencia y Universalismo) - Promoción Personal (Poder y Logro).

b. Conservación (Tradición, Conformidad y Seguridad) - Apertura al cambio (Autodirccción y Estimulación).

El hedonismo se asocia tanto con promoción personal como con apertura al cambio. Por otra parte, la asignación simultánea de importancia a valores de benevolencia, tradición y conformidad es compatible debido a que los tres tipos de valores anteponen los intereses del colectivo frente a los del individuo. De la misma manera, la asignación de importancia a valores de promoción y de apertura al cambio es compatible, pues ambos tipos de valores reflejan intereses individualistas. Es decir, anteponen la consecución de metas del individuo frente a las del colectivo.

Universalismo y seguridad incluyen valores que sirven tanto a metas del colectivo como a metas del individuo. Por lo tanto, estos tipos de valores tendrán una ubicación intermedia entre los valores colectivistas y los individualistas (Seguridad entre conformidad y poder, universalismo entre benevolencia y autodirección).

El modelo conceptual iza los sistemas de valores como estructuras integradas. Así permite generar hipótesis más coherentes y sistemáticas para el estudio de la relación entre las prioridades valorativas y otras variables actitudinales y conductuales, superando así las limitaciones de enfoques anteriores.

\section{El análisis del Espacio Mínimo (Smallest Space Analysis - SSA)}

En el proceso de construcción del instrumento para medir los valores, los datos obtenidos fueron analizados mediante la aplicación de la técnica de Guttman (1968) del Smallest Space Analysis (SSA), a la matriz de intercorrelaciones de Pearson entre los puntajes asignados a los valores, que reflejan la importancia dada por los sujetos a cada uno de los valores. 


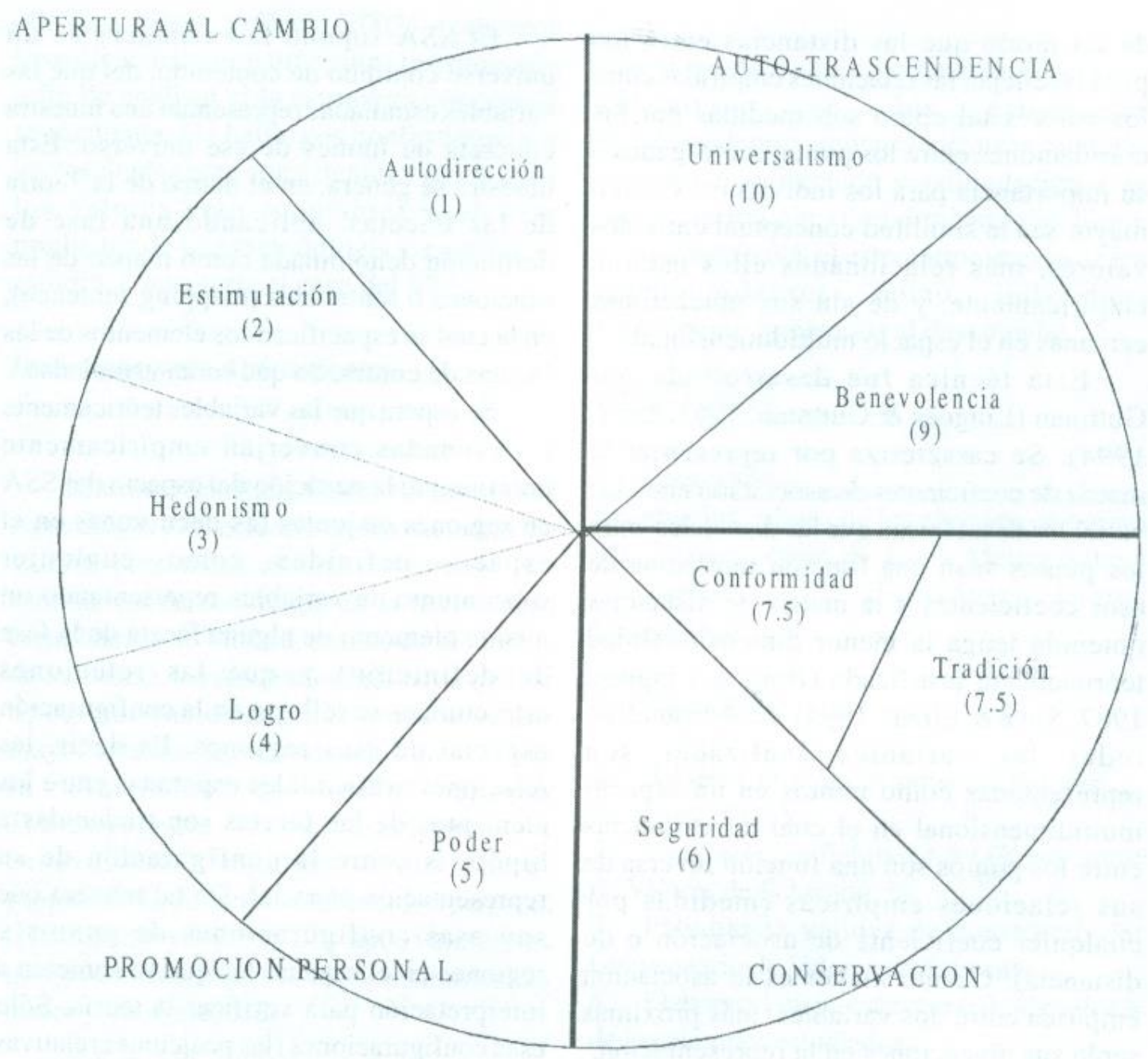

El SSA es una variedad de las técnicas de escalamiento multidimensional no métrico (AEM) para el análisis estructural de datos similares. Esta técnica representa los valores como puntos en un espacio multidimensional, de tal modo que las distancias entre los puntos reflejan las relaciones empíricas entre los valores tal como son medidas por las correlaciones entre los puntajes otorgados a su importancia para los individuos. Cuanto mayor sea la similitud conceptual entre dos valores, más relacionados ellos estarán empíricamente, y de ahí sus ubicaciones cercanas en el espacio multidimensional.

Esta técnica fue desarrollada por Guttman (Lingoes \& Guttman, 1967 Levy., 1994 ) . Se caracteriza por representar la matriz de coeficientes de asociación entre las variables de tal forma que las distancias entre los puntos sean una función monótona de esos coeficientes y la matriz de distancias obtenida tenga la menor dimensionalidad teóricamente justificada (Borg \& Lingoes, 1987; Shye \& Elizur, 1994). En éste análisis todas las variables analizadas son representadas como puntos en un espacio multidimensional en el cual las distancias entre los puntos son una función inversa de sus relaciones empíricas (medidas por cualquier coeficiente de--asociación o de distancia). Cuanto mayor es la asociación empírica entre dos variables, más próximas serán sus ubicaciones en la representación.

De acuerdo con las propuestas de Guttman, el SSA se utiliza, generalmente, en combinación con la Teoría de las Facetas (Bilsky, 2000; Borg \& Groenen, 1997; Borg \& 
Shye, 1995; Shye \& Elizur, 1994). La Teoría de las Facetas es un instrumento de análisis lógico para formalizar sistemáticamente los aspectos empíricos de la teoría, facilitando una vinculación estrecha entre el marco teórico, el diseño de los instrumentos y el análisis de los datos. Esa estrecha relación resulta muy fructífera para la investigación, en tanto obliga al investigador a hacer explícitos a priori los aspectos y la dimensionalidad del constructo disminuyendo el riesgo de interpretar ad-hoc resultados circunstanciales (Guttman \& Greenbaum, 1998).

El SSA supone la existencia de un universo continuo de contenido, del que las variables estudiadas representan una muestra concreta de ítemes de ese universo. Esta muestra se genera, en el marco de la Teoría de las Facetas, aplicando una fase de definición denominada como mapeo de las oraciones o sentencias (mapping sentence), en la cual se especifican los elementos de las facetas de contenido que serán estudiadas.

Se espera que las variables teóricamente relacionadas converjan empíricamente permitiendo la partición del espacio del SSA en regiones ,disjuntas (es decir zonas en el espacio definidas como cualquier subconjunto de variables representando un mismo elemento de alguna faceta de la fase de definición) y que las relaciones estructurales se reflejan en la configuración espacial de esas regiones. Es decir, las relaciones estructurales esperadas entre los elementos de las facetas son traducidas a hipótesis sobre la configuración de su representación espacial. De tal manera que son esas configuraciones de puntos y regiones en el espacio las que se someten a interpretación para verificar la teoría. Sólo esas configuraciones (las posiciones relativas de los puntos) tienen significado sustancial y pueden ser interpretadas, pues los ejes del SSA son arbitrarios.

El SSA proporciona una representación gráfica de los coeficientes originales como función de las distancias en la representación (diagrama de Shepard) y un «Coeficiente de Alienación que refleja el grado de ajuste de la función (entre 0 y 1; 0 indica un ajuste perfecto y un valor inferior a 0,20 es considerado adecuado). La significación del resultado depende fundamentalmente de la posibilidad de interpretar sustancialmente la representación espacial aplicando la teoría previa.

Existen estudios que han tratado de probar si la teoría de Schwartz se presenta e forma similar en diferentes culturas, es por ello que Bilsky y Koch (2002), realizaron investigaciones con diferentes instrumentos a fin de analizar si la estructura conceptual se mantenía, los hallazgos confirmaron que los resultados eran consistentes y por lo tanto los valores eran aceptables y no eran productos de una metodología específica de estudio, como sería el caso del SSA.

\section{Investigaciones Antecedentes}

Martínez Sánchez, Flores Nistal y Pérez Acosta, (1999), estudiaron los sistemas de valores personales en una muestra de 309 estudiantes de sociología de la universidad complutense de Madrid, utilizando el modelo de Schwartz, los resultados indicaron que los tipos motivacionales que presentan una mayor relevancia, son aquellos que forman la dimensión de orden superior Trascendencia (Universalismo y benevolencia), seguidos de dos valores propios de la dimensión Promoción personal (Hedonismo y logros). Los tipos valorativos menos considerados son los de estimulación, poder y conformidad.

Kornbilt, (2000), estudió los valores individuales y colectivos en una muestra de jóvenes argentinos de acuerdo a la teoría de Schwartz. Trabajó con una muestra de 750 sujetos entre 16 y 18 años, alumnos de $4^{\circ}$ año de escuelas públicas de las ciudades de Buenos Aires, Merlo, Neuquen y Bariloche. Los resultados indicaron que los jóvenes alcanzaron puntajes más altos tanto en conformidad y tradición, así como en universalismo, según la caracterización de Schwartz (1990). La autora indica que este patrón difiere en parte del encontrado en Europa occidental por el mismo Schwartz, en la medida en que en 
dicha cultura se enfatiza la autodirección a expensas de la seguridad y la preocupación por la autotrascendencia a expensas de la preocupación por la promoción personal. Además indica que el patrón valorativo hallado difiere del encontrado en los estudiantes estadounidenses, quienes alcanzan puntajes altos en autodirección y estimulación y en preocupación por el rendimiento y el logro, concordando con el estereotipo acerca de los estadounidenses como individuos fuertemente orientados al desempeño.

\section{OBJETIVOS}

\section{a. Generales}

Estudiar los valores de los alumnos de quinto año de secundaria de Lima Metropolitana pertenecientes a diferentes estratos socioeconómicos.

b. Específicos

1. Adaptar el Cuestionario de Valores de Schwartz (PVQ).

2. Evaluar la confiabilidad del Cuestionario de Valores de Schwartz.

3. Estudiar la validez de constructo del Cuestionario de Valores de Schwartz.

4. Elaborar el mapa estructural de los valores de los alumnos estudiados.

5. Evaluar los valores que presentan los alumnos del quinto año de secundaria de Lima Metropolitana de diferentes estratos socioeconómicos.

6. Analizar las diferencias en los valores es según el estrato socioeconómico al que pertenecen.

7. Identificar las diferencias significativas en los valores personales según el sexo de los alumnos.

\section{HIPÓTESIS}

H1: Los alumnos del quinto año de secundaria presentan diferencias en los valores según el estrato socioeconómico al que pertenecen.

$\mathrm{H}_{2}$ : Los alumnos varones de quinto año de secundaria presentan diferencias en los valores respecto a las alumnas mujeres.

\section{Método}

La presente investigación corresponde a un estudio de tipo descriptiva (Alarcón, 1991, Sánchez y Reyes, 2002), debido a que se trata de conocer en el aquí y el ahora como son los valores que presentan los alumnos de quinto de secundaria de Lima Metropolitana de diferentes estratos socioeconómicos.

\section{Diseño de Investigación}

El diseño de investigación, corresponde al descriptivo comparativo pues se trata de comparar los sistemas de valores personales según el estrato socioeconómico al que pertenecen los alumnos y el sexo (Alarcón, 1991; Sánchez y Reyes, 2002; Hemández, Fernández y Baptista, 2003). Su esquema es el siguiente:

$\begin{array}{ll}\mathrm{M}_{1} & \mathrm{O}_{1} \\ \mathrm{M}_{2} & \mathrm{O}_{2} \\ \mathrm{M}_{3} & \mathrm{O}_{3}\end{array}$

Donde:

$\mathrm{M}_{1}, \mathrm{M}_{2}, \mathrm{M}_{3}=$ Muestras de investigación de los estratos socioeconómicos estudiados.

$\mathrm{O}_{1}, \mathrm{O}_{2}, \mathrm{O}_{3}=$ Observaciones de las variables estudiadas. 


\section{Participantes}

El universo de investigación estuvo conformado por los 79.128 alumnos matriculados de acuerdo a las estadísticas reportadas por el Ministerio de Educación el año 200 1. El tamaño de la muestra fue establecido a través del programa informático SOTAM, el cual determinó que con un margen de error de 5\% Y un nivel de confianza del $99.9 \%$, correspondió un tamaño de muestra de 1197 casos.

Para la obtención de la muestra se aplicó un procedimiento de muestreo probabilístico estratificado, clasificando a los alumnos de acuerdo al sexo, tipo de colegio de procedencia y la USE a la que pertenecían.

\section{Composición de la muestra}

La muestra estuvo constituida por 1197 alumnos (ver tabla 1), con edades que fluctuaban entre los 14 y los 18 años, siendo la mayor cantidad la que correspondió a los que tuvieron 16 años (40,80\%). También se observo que el 50,90\% fueron mujeres y el $49,10 \%$ fueron varones.

Respecto al colegio de procedencia existieron más alumnos pertenecientes a colegios estatales $(49,29 \%)$ que a colegios particulares $(50,71 \%)$. La mayoría de alumnos eran de Lima $(71,40 \%)$. Con relación al estrato socioeconómico se observó que el $37.60 \%$ eran del estrato bajo, el $41,60 \%$ del estrato medio y el $20.80 \%$ del estrato alto. También se encontró que la gran mayoría vivía con su familia $(97,80 \%)$.

Tabla 1 Composición general de la muestra de alumnos evaluados

\begin{tabular}{|l|l|l|l|}
\hline & Variable & F. & $\%$ \\
\hline \multirow{4}{*}{ Edad } & 14 años & 63 & 5.20 \\
& 15 años & 495 & 41.40 \\
& 16 años & 489 & 40.80 \\
17 años & 100 & 8.40 \\
& 50 & 4.20 \\
\hline \multirow{2}{*}{ Sexo } & 18 años & 588 & 49.10 \\
& Varon & 609 & 50.90 \\
\hline \multirow{2}{*}{ Tipo de colegio } & Mujer & 590 & 49.29 \\
& Estatal & 607 & 50.71 \\
\hline Estrato & No estatal & 450 & 37.60 \\
socioeconomico & Bajo & 498 & 41.60 \\
& Alto & 249 & 20.80 \\
\hline
\end{tabular}

$\mathrm{N}=1197$ 


\section{Variables}

\section{Variables independientes asignadas}

- Estrato socioeconómico: Bajo, Medio y Alto.

- Sexo de los alumnos: Varón y Mujer.

\section{Variable dependiente:}

- Valores: evaluado a través de del cuestionario de valores de Schwartz (PVQ)

Variables controladas:

- Nivel Educativo: Alumnos de 5to. año de secundaria.

- Edad, los alumnos podían tener entre 14 y 18 años.

\section{Instrumentos}

\section{Cuestionario de Valores de Schwartz ( PVQ)}

El Cuestionario de Valores de Schwartz (Schwartz, 2000), es un instrumento que fue desarrollado con la finalidad de evaluar de una manera más concreta y menos cognitiva los valores propuestos por la teoría de Schwartz, de tal manera que se pueda utilizar en cualquier tipo de población, a la vez que permitiera servir de instrumento que de forma independiente permita evaluar el contenido y la estructura teórica de los valores según Schwartz.

El PVQ incluye 40 enunciados cortos que aluden a 29 diferentes tipos de personas, describiéndose a cada una de ellas sus ideas, creencias y aspiraciones que de forma implícita permiten analizar la importancia del valor evaluado. Los valores estudiados con el PVQ son:

- Hedonismo.

- Seguridad.

- Poder.

- Logro.

- Autodirección.

- Conformidad.

- Universalismo.

- Estimulación.

- Tradición.

- Benevolencia.

Para la aplicación del instrumento la consigna utilizada indica que se describen brevemente algunas personas, y que indique en cada descripción cuánto se parece o no a cada una de ellas. Para responder se debe utilizar la siguiente escala:

- Se parece mucho a mí

- Es como yo.

- Se parece algo a mí.

- Se parece poco a mí.

- No se parece a mí.

- No se parece nada a mí.

Los resultados empíricos obtenidos evidencian que la prueba presenta con fiabilidad por consistencia interna y validez convergente, observándose también que existe con las variables consideradas para analizar la validez ecológica del modelo, (Schwartz, Gila, 
Lehmann, Burgess, Harris y Owen, 2001)

Debido a la necesidad de adaptar el instrumento a nuestro medio, se realizaron los siguientes análisis psicométricos:

a) Traducción de la versión Inglesa al castellano.

b) Análisis de la adecuación lingüística de los ítemes.

c) Aplicación de del instrumento

d) Análisis de los Ítemes.

e) Estudio de la validez de constructo.

f) Estudio de la confiabilidad.

g) Construcción del mapa estructural de acuerdo al Análisis de Escalamiento Multidimensional (AEM), en base a la técnica de Guttman del Smallest Space Analysis (SSA).

\section{Índice de Estrato socioeconómico}

Para medir la pertenencia a un determinado socioeconómico se construyó un indicador específico que permitiera identificar el estrato de procedencia de los sujetos, utilizándose como esquema el estudio sobre niveles socioeconómicos efectuados por Apoyo Opinión y Mercado S. A. (1999) Seleccionándose como criterios de calificación los siguientes aspectos:

a.Poder adquisitivo de la familia, expresado en el ingreso familiar promedio mensual.

b.Nivel educativo alcanzado por ambos padres.

c. Zona de residencia de la familia.

d.Acceso a bienes, identificado por el uso de electrodomésticos.

e. Acceso a servicios.

f. Materiales predominantes utilizados en la construcción de la casa

g.Número de miembros de la familia.

h.Número de habitaciones de la casa.

i.. Tipo de vivienda.

Cada pregunta fue calificada en una escala de 5 puntos según la predominancia del aspecto evaluado. Los datos permitieron conformar una escala de puntajes tipo Likert, los cuales alcanzaron un coeficiente Alfa de Cronbach de 0.86 y el análisis factorial exploratorio arrojó la existencia de un solo factor, lo que permitió concluir que el Índice presentó Validez de Constructo.

Los puntajes de cortes para realizar la clasificación de los estratos socioeconómicos se efectuaron contrastando los puntajes alcanzados en el Índice con la clasificación generada con el sistema del Grupo Apoyo, notándose que presentó validez convergente.

\section{RESULTADOS}

\section{Análisis Psicométricos}

El análisis psicométrico se realizó a través de la correlación ítem-test corregida (ritc) y el coeficiente de confiabilidad por consistencias Alfa de Cronbach (Muñiz, 1996; 1994). 
Tabla 2. Análisis de ítems y confiabilidad de los componentes del cuestionario de valores de Schwartz (PVQ)

\begin{tabular}{|c|c|c|c|}
\hline Items & M & D.E. & ritc \\
\hline \multicolumn{4}{|l|}{ Conformidad } \\
\hline $\begin{array}{l}7 \\
16 \\
28 \\
36 \\
\end{array}$ & $\begin{array}{l}3,44 \\
4,11 \\
4,59 \\
4,45 \\
\end{array}$ & $\begin{array}{l}1,31 \\
1,37 \\
1,29 \\
1,28 \\
\end{array}$ & $\begin{array}{l}0,45^{*} \\
0,49 * \\
0,48^{*} \\
0,46 *\end{array}$ \\
\hline & \multicolumn{3}{|c|}{ Alfa de Cronbach $=0,70 *$} \\
\hline $\begin{array}{l}\text { Tradición } \\
9 \\
20 \\
25 \\
38 \\
\end{array}$ & $\begin{array}{l}3,39 \\
3,63 \\
3,63 \\
4,33 \\
\end{array}$ & $\begin{array}{l}1,51 \\
1,41 \\
1,57 \\
1,25 \\
\end{array}$ & $\begin{array}{l}0,23 \\
0,22 \\
0,35 \\
0,24 \\
\end{array}$ \\
\hline & \multicolumn{3}{|c|}{ Alfa de Cronbach $=0,75 *$} \\
\hline \multicolumn{4}{|l|}{ Benevolencia } \\
\hline $\begin{array}{l}12 \\
18 \\
27 \\
33 \\
\end{array}$ & $\begin{array}{l}4,63 \\
4,69 \\
4,61 \\
4,22 \\
\end{array}$ & $\begin{array}{l}1,24 \\
1,37 \\
1,24 \\
1,37\end{array}$ & $\begin{array}{l}0,68 * \\
0,56 * \\
0,68 * \\
0,41 *\end{array}$ \\
\hline & \multicolumn{3}{|c|}{ Alfa de Cronbach $=0,77 *$} \\
\hline \multicolumn{4}{|l|}{ Universalismo } \\
\hline \begin{tabular}{|l|}
3 \\
8 \\
19 \\
26 \\
29 \\
40 \\
\end{tabular} & $\begin{array}{l}4,80 \\
4,37 \\
4,52 \\
4,61 \\
4,72 \\
4,35\end{array}$ & $\begin{array}{l}1,39 \\
1,32 \\
1,32 \\
1,33 \\
1,30 \\
1,37\end{array}$ & $\begin{array}{l}0.69 * \\
0,49 * \\
0,68 * \\
0,69 * \\
0,72 * \\
0,57 *\end{array}$ \\
\hline & \multicolumn{3}{|c|}{ Alfa de Cronbach $=0,85 *$} \\
\hline \begin{tabular}{|l|} 
Autodirección \\
1 \\
11 \\
22 \\
34
\end{tabular} & $\begin{array}{l}4,51 \\
4,80 \\
4,61 \\
4,57\end{array}$ & $\begin{array}{l}1,24 \\
1,34 \\
1,23 \\
1,27\end{array}$ & $\begin{array}{l}0,58 * \\
0,60 * \\
0,57 * \\
0,56 *\end{array}$ \\
\hline 34 & \multicolumn{3}{|c|}{ Alfa de Cronbach $=0,77 *$} \\
\hline
\end{tabular}


Los resultados del análisis psicométrico presentado en la Tabla $\mathrm{N}^{\circ} 2$, indicaron que los valores de: Conformidad, Tradición, Benevolencia, Universalismo y Autodirección presentaron correlaciones Ítem-test corregidas para cada valor que superan el criterio establecido por Kline (1995) de 0.20 y además se apreció que los coeficientes alfa de Cronbach fluctuaron entre 0.70 y 0.85 , lo que indicaría que los valores evaluados son confiables.

Tabla 3. Análisis de Ítemes v confiabilidad de los comnonentes del de Valores de Schwartz (PVQ).

\begin{tabular}{|c|c|c|c|}
\hline Itemes & $\mathbf{M}$ & D.E. & ritc \\
\hline \multicolumn{4}{|c|}{ Estimulación } \\
\hline 6 & 4,56 & 1,27 & 0,53 \\
\hline 15 & 3,97 & 1,38 & 0,43 \\
\hline 30 & 4,57 & 1,37 & 0,53 \\
\hline \multicolumn{4}{|c|}{ Alfa de Cronbach $=0,72 *$} \\
\hline Hedonismo & 429 & 138 & 052 \\
\hline 26 & 4,38 & 1,29 & 0,59 \\
\hline 37 & 4,68 & 1,28 & 0,56 \\
\hline \multicolumn{4}{|c|}{ Alfa de Cronbach $=0,74 *$} \\
\hline \multicolumn{4}{|l|}{ Logro } \\
\hline 4 & 4,33 & 1,33 & 0,50 \\
\hline 13 & 4,19 & 1,31 & 0,57 \\
\hline 24 & 4,03 & 1,56 & 0,31 \\
\hline 32 & 4,69 & & \\
\hline 1,30 & & $0,35 *$ & \\
\hline & \multicolumn{3}{|c|}{ Alfa de Cronbach $=0,79 *$} \\
\hline Poder & & & \\
\hline 2 & 3,33 & $\mathrm{~J}, 42$ & '0,37 \\
\hline 17 & 3,40 & 1,39 & 0,44 \\
\hline \multirow[t]{2}{*}{39} & 4,13 & 1,35 & 0,39 \\
\hline & \multicolumn{3}{|c|}{ Alfa de Cronbach $=0,75 *$} \\
\hline \multicolumn{4}{|l|}{ Seguridad } \\
\hline 5 & 4,48 & 1,36 & 0,50 \\
\hline 14 & 4,35 & 1,39 & 0,52 \\
\hline 21 & 4,35 & 1,38 & $0,49 *$ \\
\hline 31 & 4,24 & 1,36 & 0,47 \\
\hline 35 & 4,39 & 1,34 & 0,54 \\
\hline \multicolumn{4}{|c|}{ Alfa de Cronbach $=0,78 *$} \\
\hline
\end{tabular}

Con relación con los hallazgos alcanzados en el análisis psicométrico (ver Tabla 3), indicaron que los valores de estimulación, hedonismo, logro, poder y seguridad presentaron correlaciones ítem-test corregidas adecuadas y además se apreció que los coeficientes Alfa de Cronbach puntuaron entre 0.72 y 0.79 , lo que indicaría que los valores evaluados son confiables. 
Tabla 4. Validez de Constructo a través del Análisis Factorial Exploratorio del Cuestionario de Valores de Schwartz (PVQ).

\begin{tabular}{|l|l|l|l|l|}
\hline Valores & $\mathrm{M}$ & D.E. & Factor 1 & Factor 2 \\
\hline Conformidad & 4,15 & 0,89 & 0,78 & \\
Tradición & 3,75 & 0,84 & 0,69 & \\
Benevolencia & 4,54 & 1,01 & 0,77 & \\
Universalismo & 4,56 & 1,02 & 0,81 & \\
Autodirección & 4,63 & 0,98 & & 0,60 \\
Estimulación & 4,37 & 1,04 & & 0,66 \\
Hedonismo & 4,41 & 1,06 & & 0,72 \\
Logro & 4,31 & 0,96 & & 0,73 \\
Poder & 3,62 & 1,02 & & 0,70 \\
Seguridad & 4,36 & 0,96 & 0,71 & \\
\hline Varianza Explicada & & & $51,20 \%$ & 24,90 \\
\hline
\end{tabular}

Medida de Adecuación del Muestreo De Kaiser-Meyer-Olkin = 0,96 Test de esfericidad de Bartlett $=6828,72 * * *$

$* * * \mathrm{p}<, 001 \mathrm{~N}=1197$

Los resultados alcanzados en el análisis factorial exploratorio, realizado utilizando el método de los componentes principales y el método de rotación Oblimin, permitieron denotar que la medida de adecuación del muestreo de Kaiser-MeyerOlkin alcanzó un valor de 0,96 el cual puede considerarse como adecuado, mientras que el test de esfericidad de Bartlett presenta un valor que es significativo $(B=6828,72 ; \mathrm{P}<.001)$, lo que nos indicó que los coeficientes de correlación entre los ítemes fueron lo suficientemente elevados como para continuar con el análisis factorial. Estos hallazgos permitieron corroborar la pertinencia de utilizar el análisis factorial en los datos del estudio.

Los resultados del análisis indicaron que existieron dos factores que permitieron explicar el 76, I 0\% de la varianza total. El primer factor explicó el 51,20 \% de la varianza total, e incluyó a los valores de tradición, conformidad y seguridad (que constituyeron la dimensión de conservación), así como por benevolencia y universalismo (que constituyó la dimensión de trascendencia).

El segundo factor explicó el 24,90 \% de la varianza total, estuvo conformado por autodirección y estimulación (que constituyeron la dimensión de apertura al cambio) y por hedonismo, logro, poder (que conformaron la dimensión de promoción personal).

Los resultados alcanzados permitieron concluir que el Cuestionario de Valores de Schwartz presentó validez de constructo. 
Tabla 5. Resultados del Análisis de Escalamiento Multidimensional del SSA del PQV de Schwartz.

\begin{tabular}{|l|l|c|}
\hline & \multicolumn{2}{|c|}{ Configuración Final } \\
\hline Valores & Dimensión 1 & Dimensión 2 \\
\hline Conformidad & 0.75 & 0.33 \\
Tradición & 0.86 & -1.33 \\
Benevolencia & 0.57 & -0.31 \\
Universalismo & 0.54 & -0.28 \\
Autodirección & 0.08 & -0.48 \\
Estimulación & -0.21 & -0.75 \\
Hedonismo & -0.37 & -0.72 \\
Logro & -0.75 & 0.05 \\
Poder & -1.77 & 0.85 \\
Seguridad & 0.30 & 0,08 \\
\hline Rango del Stress DHAT & 0.11 \\
Stress DHAT & 0.03 \\
Coeficiente de alimentación DSTAR & 0.06 \\
Stress basado en la aproximación a los datos aleatorios 0.15 \\
\hline
\end{tabular}

El análisis de Escalamiento Multidimensional, utilizando la técnica de Guttman del SSA (Smallest Space Analysis) fue efectuado a través de los programas MINISSA (MDS(X), 2001), PERMAP 11.0 (Heady \& Lucas, 2003) y PAP-FACETS (Hammond, 1999). Los resultado presentados en la Tabla $\mathrm{N}^{\circ}$, permitieron apreciar que el mapa estructural de dos dimensiones fue eficiente, pues se encontró que las 2 dimensiones propuestas permitieron obtener valores consistentes tal como 10 indicaron el Rango del Stress DHAT $(0,11)$, el Stress DHAT $(0,03)$, el Coeficiente de Alienación DSTAR $(0,06)$ y la medida del Stress basado en la aproximación a los datos aleatorios $(0,15)$.

En lo que concierne a la revisión del gráfico del análisis estructural del SSA (Ver Gráfico 2) de la propuesta del modelo teórico de Schwartz, se encontró que la ubicación geográfica de los valores de conformidad, tradición, seguridad, poder, logro, hedonismo, estimulación, autodirección, correspondió con los valores encontrado en los estudios transculturales, en tanto que universalismo y benevolencia, presentaron una ligera modificación, pues se observó que el universalismo, se ubicó en el lugar de benevolencia y benevolencia a su vez ocupó el Jugar de universalismo. Notándose que la correlación entre la ubicación del modelo original y el modelo observado en los alumnos de 5to. grado de secundaria fue 0,98 , por lo cual fue clasificado como positiva alta y significativa a $p<, 001$, hallazgo corroboró la adecuación del modelo teórico de Schwartz en nuestro contexto.

\section{Análisis descriptivos}

Los resultados del análisis de la bondad de ajuste a la curva normal, fueron realizados a través de la prueba de Kolmogorov-Smirnov (ver Tabla $\mathrm{N}^{\circ}$ 6), e indicaron que en todos los valores se obtuvieron estadísticos $\mathrm{K}-\mathrm{S} \mathrm{Z}$ que no son significativos, por lo que se pudo concluir que las distribuciones de los valores analizados se aproximaron adecuadamente a la distribución normal. Es por ello que se utilizaron contrastes estadísticos paramétricos en el análisis de los datos de la investigación (Siegel y Castellan, 1995).

Adicionalmente se observó que el orden establecido de acuerdo a la puntuación recibida 
permitió identificar que en la muestra en general los valores mejor calificados fueron: autodirección $(\mathrm{M}=4,63$ D.E: $=0,98)$, universalismo $(\mathrm{M}=4,56$ D.E.= 1,02) Y benevolencia $(M=4,54$ D.E: $=1,01)$, en tanto que los menos calificados correspondieron a: conformidad $(M=4,15$ D.E: $=0,89)$, tradición $(M=3,75$ D.E. $=0,84) Y$ benevolencia $(M=$ 3,62 D.E: $=1,02)$.

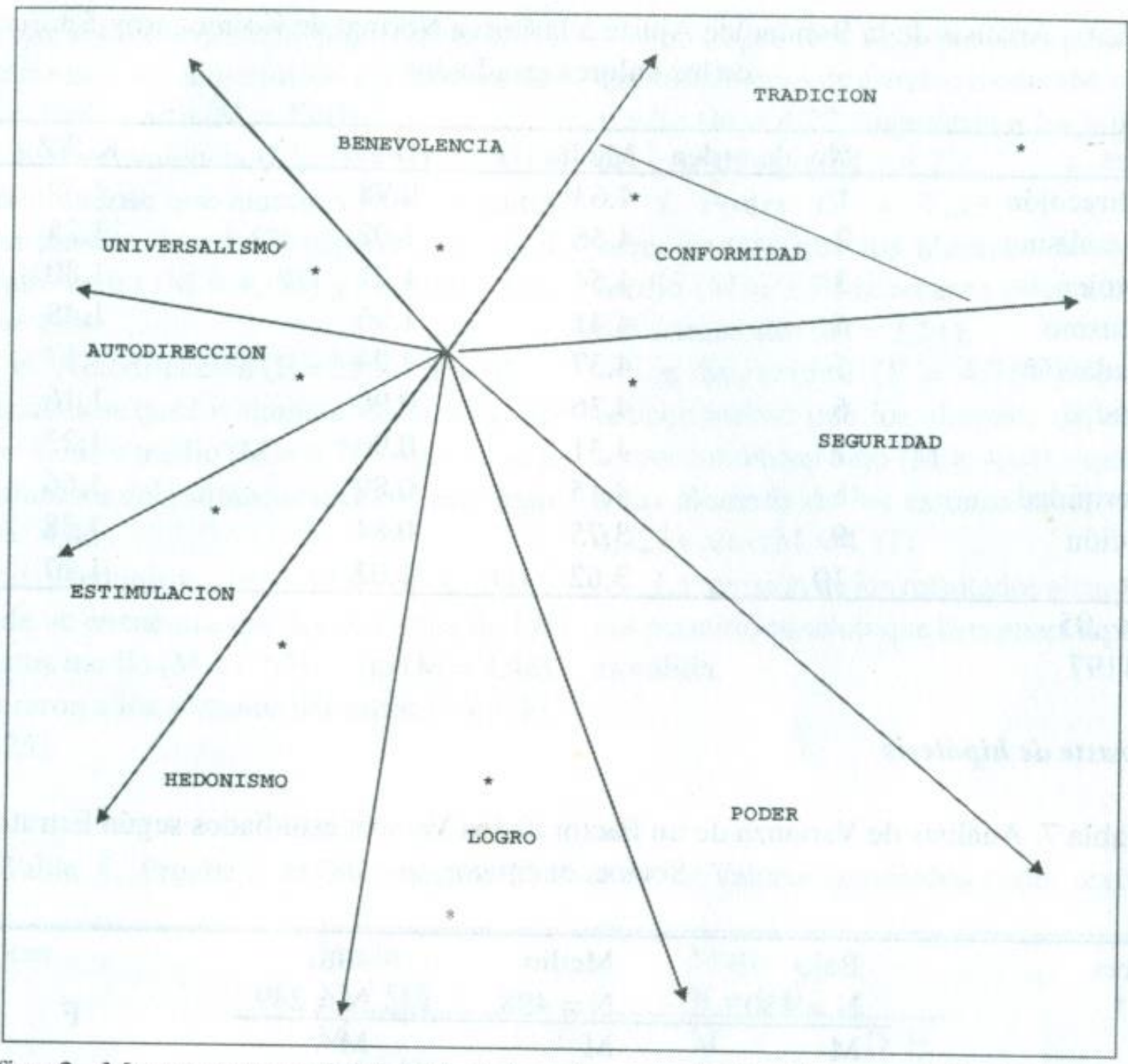

Grafico 2. Mapa estructural del SSA de los alumnos de 5to. de secundaria de Lima Metropolitana 
Tabla 6. Análisis de la Bondad de Ajuste a la Curva Normal de Kolmogorov-Smirnov de los valores estudiados.

\begin{tabular}{|l|l|l|l|l|}
\hline Valores & $\begin{array}{l}\mathrm{N}^{\circ} \text { de } \\
\text { orden }\end{array}$ & Media & D.E & K-SZ \\
\hline Autodirección & 1 & 4,63 & 0.98 & 1,39 \\
Universalismo & 2 & 4,56 & 1,02 & 1,25 \\
Benevolencia & 3 & 4,54 & 1,01 & 1,80 \\
Hedonismo & 4 & 4,41 & 1,06 & 1,48 \\
Estimulación & 5 & 4,37 & 1,04 & 1,17 \\
Seguridad & 6 & 4,36 & 0,96 & 1,16 \\
Logro & 7 & 4,31 & 0,96 & 1,23 \\
Conformidad & 8 & 4,15 & 0,89 & 1,66 \\
Tradición & 9 & 3,75 & 0,84 & 1,38 \\
Poder & 10 & 3,62 & 1,02 & 1,27 \\
\hline
\end{tabular}

$* \mathrm{p}<, 05$

$\mathrm{N}=1197$

Contraste de hipótesis

Tabla 7. Análisis de Varianza de un Factor de los Valores estudiados según Estrato Socioeconómico.

\begin{tabular}{|c|c|c|c|c|}
\hline valores & $\begin{array}{l}\text { Bajo } \\
\mathrm{N}=450\end{array}$ & $\begin{array}{l}\text { Medio } \\
\mathrm{N}=498\end{array}$ & $\begin{array}{l}\text { Alto } \\
\mathrm{N}=249\end{array}$ & $\mathrm{~F}$ \\
\hline & $\mathrm{M}$ & $\mathrm{M}$ & $\bar{M}$ & \\
\hline Conformidad & $4.34 \mathrm{ab}$ & $4,04^{\mathrm{a}}$ & $4,01 \mathrm{~b}$ & 29,12 \\
\hline Tradición & $4.01 \mathrm{ab}$ & $3,58^{\mathrm{a}}$ & $3,60 b$ & $3.78 * * *$ \\
\hline Benevolencia & $4,44 a b$ & $4,56 \mathrm{ac}$ & $4,67 b c$ & $10.83 * * *$ \\
\hline Universalismo & 4,56 & 4,53 & 4,62 & 2.46 \\
\hline Autodirección & $4,46 a b$ & $4,70 \mathrm{a}$ & $4,75 b$ & $25.12 * * *$ \\
\hline Estimulación & 4,30 & 4,40 & 4,43 & 2.79 \\
\hline Hedonismo & $4,25 \mathrm{ab}$ & $4,53 \mathrm{a}$ & $4,48 b$ & $13.06 * * *$ \\
\hline Logro & $04,22 \mathrm{a}$ & $4,41 \mathrm{ab}$ & $4,28 b$ & $7.53 *$ \\
\hline Poder & 3,59 & $3,70 \mathrm{a}$ & $3,51^{\mathrm{a}}$ & $3.39 *$ \\
\hline Seguridad & $4,44 a b$ & $4,32 \mathrm{a}$ & $4,31 b$ & $4.78 *$ \\
\hline
\end{tabular}

Diferencias significativas por pares a través de la prueba de Scheffé: $a, b, c$

En el análisis de la primera hipótesis $\mathrm{H}_{\mathrm{J}}$, que propuso que existen diferencias en los valores según el estrato socioeconómico, los resultados del análisis de varianza de un factor de los valores aplicando el factor de corrección de puntajes propuesto por Schwartz (Schwartz, Melech, Lehmann, Burgess, Harris, \& Owens, 2001) indicaron que existieron diferencias estadísticas significativas según el estrato socioeconómico en los siguientes valores:

a. Tradición $(\mathrm{F}=3,78 \mathrm{P}<, 001)$, notándose que los alumnos pertenecientes al estrato socioeconómico bajo $(\mathrm{M}=4,01)$ superaron a los alumnos de estratos medio $(\mathrm{M}=$ $3,58)$ y alto $(M=3,60)$. 
b. Benevolencia $(\mathrm{F}=10,83 \mathrm{p}<, 001)$, encontrándose que alumnos pertenecientes al estrato alto $(M=4,67)$ superaron a los del estrato medio $(M=4,56)$ y el estrato bajo $(M=$ 4,44).

c. Autodirección $(\mathrm{F}=25,12 \mathrm{p}<, 001)$, apreciándose que los alumnos de estratos alto $(\mathrm{M}=4,75) \mathrm{Y}$ medio $(\mathrm{M}=4,70)$, superaron a los alumnos del estrato socioeconómico bajo $(\mathrm{M}=4,46)$.

d. Hedonismo $(\mathrm{F}=13,06 \mathrm{p}<, 001)$, donde se encuentra que los alumnos de los estratos medio $(M=4,53) \mathrm{Y}$ alto $(\mathrm{M}=4,48)$ superaron a los alumnos del estrato bajo $(\mathrm{M}=4,25)$.

e. Logro $(\mathrm{F}=7,53 \mathrm{p}<, 05)$, notándose que los alumnos de estrí(tos medio $(\mathrm{M}=4,41)$ y Alto $(M=4,28)$ superaron a los alumnos del estrato bajo $(M=4,22)$.

f. Poder $(\mathrm{F}=7,53 \mathrm{P}<, 05)$, apreciándose que los alumnos del estrato medio $(\mathrm{M}=3,70)$ superaron a los alumnos del estrato alto $(\mathrm{M}=3,51)$.

g. Seguridad $(F=4,78 \mathrm{P}<, 05)$, encontrándose que los alumnos del estrato socioeconómico bajo $(\mathrm{M}=4,44)$ superaron a los alumnos de los estratos medio $(\mathrm{M}=4,32)$ $\mathrm{Y}$ alto $(\mathrm{M}=4,31)$

La revisión de los resultados alcanzados nos permitió concluir que la primera hipótesis es válida.

Tabla 8. Prueba $\mathrm{Z}$ de diferencia de Medias de los Valores estudiados según sexo.

\begin{tabular}{|l|ll|ll|c|}
\hline Valores & Varón & & \multicolumn{2}{|l|}{ Mujer } & \\
& $\mathrm{N}=588$ & & $\mathrm{~N}=609$ & \\
& $\mathrm{M}$ & D.E. & $\mathrm{M}$ & $\mathrm{D} . \mathrm{E}$. & $\mathrm{Z}$ \\
\hline Conformidad & 4,30 & 0,87 & 4,17 & 0,91 & $-1,20$ \\
Tradición & 3,79 & 0,84 & 3,71 & 0,84 & 3,07 \\
Benevolencia & 4,46 & 0,95 & 4,62 & 1,06 & $-20,06$ \\
U niversalismo & 4,52 & 0,98 & 4,60 & 1,05 & $-4,50 *$ \\
Autodirección & 4,62 & 0,95 & 4,63 & 1,01 & $-0,32$ \\
Estimulación & 4,44 & 1,04 & 4,38 & 4,05 & 1,60 \\
Hedonismo & 4,50 & 1,01 & 4,33 & 1,09 & 1,43 \\
Logro & 4,35 & 0,94 & 4,27 & 0,97 & $4,71 *$ \\
Poder & 3,78 & 0,96 & 3,49 & 1,05 & $22,20 * * *$ \\
Segurid & 4,30 & 0,95 & 4,42 & 0,97 & $-14,18$ \\
\hline
\end{tabular}

$* \mathrm{P}<, 05 * * \mathrm{P}<, 01 * * * \mathrm{P}<, 001 \mathrm{~N}=1197$

Con relación a la segunda hipótesis $\mathrm{H}_{2}$, en la cual se indicó que existen diferencias en los valores según el sexo del alumno, el análisis realizado a través de la prueba $\mathrm{Z}$ de diferencia de medias, presentado en la Tabla $N^{\circ} 8$, permitió observar que existieron diferencias estadísticas significativas a favor de las mujeres en benevolencia $(Z=-20,06, P$ $<, 001)$ universalismo $(Z=-4,50 \mathrm{P}<, 05) \mathrm{Y}$ seguridad $(\mathrm{Z}=-14,18, \mathrm{P}<, 001)$. En tanto que los varones presentaron puntajes significativos en logro $(\mathrm{Z}=4,71 \mathrm{P}<, 05)$ Y poder $(\mathrm{Z}=$ $22,20 \mathrm{P}<, 001)$. Estos hallazgos permitieron concluir que la segunda hipótesis fue válida. 


\section{Análisis complementarios}

A continuación se exponen los resultados complementarios obtenidos en el análisis de los valores.

Tabla 9. Organización Jerárquica de los Valores en cada estrato socioeconómico.

\begin{tabular}{|c|ll|ll|lc|}
\hline \multirow{2}{*}{ Orden } & \multicolumn{2}{|c|}{ Bajo } & \multicolumn{2}{c|}{ Medio } & \multicolumn{2}{c|}{ Alto } \\
\cline { 2 - 7 } & Valor & $\mathrm{M}$ & Valor & $\mathrm{M}$ & Valor & $\mathrm{M}$ \\
\hline 1 & Universalismo & 4,56 & Autodirección & 4,70 & Autodirección & 4,75 \\
2 & Autodirección & 4,46 & Benevolencia & 4,56 & Benevolencia & 4,67 \\
3 & Benevolencia & 4,44 & Universalismo & 4,53 & Universalismo & 4,62 \\
4 & Seguridad & 4,44 & Hedonismo & 4,53 & Hedonismo & 4,48 \\
5 & Conformidad & 4,34 & Logro & 4,41 & Estimulación & 4,43 \\
6 & Estimulación & 4,30 & Estimulación & 4,40 & Seguridad & 4,31 \\
7 & Hedonismo & 4,25 & Seguridad & 432 & Logro & 4,28 \\
8 & Logro & 4,22 & Conformidad & 4,04 & Conformidad & 4,01 \\
9 & Tradición & 4,01 & Poder & 3,70 & Tradición & 3,60 \\
10 & Poder & 3,59 & Tradición & 3,58 & Poder & 3,51 \\
\hline
\end{tabular}

La revisión del análisis jerárquico de los valores de acuerdo a cado estrato socioeconómico (Ver Tabla 9), permitió observar que en los tres grupos predominaron los tres primeros valores (Universalismo, autodirección y benevolencia), pero se observó que mientras en el estrato bajo predominó el universalismo, en los estratos medio y alto predominó la autodirección.

Mientras que con relación a los valores menos calificados, se apreció que los grupos medio y alto presentaron valores similares (Conformidad, tradición y poder), en tanto que en el grupo bajo en lugar de la conformidad se presentó el logro.

Tabla 10. Organización Jerárquica de los Valores

\begin{tabular}{|c|lc|lr|}
\hline Orden & \multicolumn{2}{|c|}{ Varón } & \multicolumn{2}{c|}{ Mujer } \\
\hline & Valor & M & Valor & M \\
\hline 1 & Autodirección & 4,62 & Autodirección & 4,6 \\
2 & Universalismo & 4,52 & Benevolencia & 4,6 \\
3 & Hedonismo & 4,50 & Universalismo & 4,6 \\
4 & Benevolencia & 4,46 & Seguridad & 4,4 \\
5 & Estimulación & 4,44 & Estimulación & 4,3 \\
6 & Logro & 4,35 & Hedonismo & 4,3 \\
7 & Conformidad & 4,30 & Logro & 4,2 \\
8 & Seguridad & 4,30 & Conformidad & 4,1 \\
9 & Tradición & 3,79 & Tradición & 3,7 \\
10 & Poder & 3,78 & Poder & 3,4 \\
\hline
\end{tabular}

En lo que respecta a la revisión del análisis jerárquico de los valores de acuerdo al sexo, en la Tabla 10), se encontró que tanto los hombres como las mujeres presentaron la autodirección como valor predominante, siendo otro valor común el universalismo, aunque los varones presentaron el hedonismo y en cambio las mujeres puntuaron más la benevolencia. Con relación a los valores menos calificados, se apreció que ambos grupos presentaron valores similares (Tradición y poder), en tanto que en el grupo de varones también se incluyó la seguridad mientras en las mujeres se pareció la conformidad

Con la finalidad de identificar la existencia de patrones similares de responder a los valores, 
se efectuó un análisis de Cluster (Hair, Anderson, Tatham \& Black, 1999). Los resultados obtenidos en la Tabla 11, indicaron que existían grupos de sujetos con perfiles diferenciados, notándose que las comparaciones de los valores entre los grupos presentaron diferencias estadísticas significativas

\section{Tabla 11. Análisis de Cluster de lo valores estudiados}

\begin{tabular}{|c|c|c|c|c|}
\hline Valores & $\begin{array}{l}\text { Gruno } 1 \\
\mathrm{~N}=510\end{array}$ & $\begin{array}{l}\text { Gruno } 2 \\
\mathrm{~N}=533\end{array}$ & $\begin{array}{l}\text { Gruno } 3 \\
\mathrm{~N}=153^{3}\end{array}$ & $\mathrm{~F}$ \\
\hline & $\mathbf{M}$ & $\mathrm{M}$ & $\mathbf{M}$ & \\
\hline Conformidad & 3,95 & 4,67 & 2,97 & $397,57 * * *$ \\
\hline Tradición & 3,62 & 4,03 & 3,18 & $80,35 * * *$ \\
\hline Benevolencia & 4,48 & $5, .12$ & 2,72 & $782,07 * * *$ \\
\hline Universalismo & 4,53 & 5,15 & 2,62 & $947,63 * * *$ \\
\hline Autodirección & 4,57 & 5,20 & 2,75 & $1000, .19^{\prime} * *$ \\
\hline Estimulación & 4,26 & 4,98 . & $2,6^{\prime} 1:$ & $6,32,75 * * *$ \\
\hline Hedonismo & 4,25 & 5,03 & $2,8^{\prime} \mathrm{U}$ & 502',28: **"* \\
\hline Logro & 3,98 & 4,97 & 3,12 & $499,44 * * *$ \\
\hline Poder & 3,14 & 4,06 & 3,72 & $131,02 * * *$ \\
\hline Seguridad & 4,13 & 5,00 & 2,91 & $643,50 * * *$ \\
\hline
\end{tabular}

Tal como se puede apreciar en el gráfico 3, se observó que en el contraste de todos los valores entre los grupos identificados, existieron diferencias estadísticas significativas.

El análisis pormenorizado permitió denotar que el grupo 1 correspondió a los alumnos que presentaron valores intermedios en todos los casos y constituyeron 510 casos. El grupo 2 , incluyó a los alumnos con puntajes más elevados en todos los valores y estuvieron conformados por 533 alumnos, mientras que el grupo 3 incluyó a los alumnos con bajas puntuaciones en los valores y estuvo conformado por 153 casos, este grupo podría considerarse como de riesgo.

\section{Gráfico 3. Perfil de Valores de Schwartz por grupo}

Con la finalidad de corroborar la existencia de los grupos identificados se desarrolló un análisis discriminante (ver Tabla 12), el cual corroboró la existencia de los perfiles de los grupos observados, notándose la existencia de dos Funciones Canónicas que alcanzaron una correlación Canónica de 0,91 y que permitieron explicar el 94,21 \% de la varianza total.

El análisis de la primera función canónica indicó que estuvo asociada más a los valores de: conformidad, tradición, benevolencia, universalismo, autodirección, estimulación, hedonismo y seguridad, en tanto que la segundas función discriminante correlacionó positivamente con los valores de logro y poder.

Adicionalmente el análisis de la capacidad de discriminación de los grupos identificados, en la Tabla 13, permitió denotar que entre el grupo predicho y el grupo real existió un 98,96\% de concordancia, lo cual permitió corroborar la pertinencia de la clasificación de los grupos de alumnos de acuerdo al perfil de valores que presentaron. 
Tabla 12. Análisis Discriminante de los grupos identificados.

\begin{tabular}{|l|c|l|}
\hline Valores & Función Canónica 1 & Función Canónica 2 \\
\hline Conformidad & $0,37^{*}$ & 0,17 \\
Tradición & $0,16^{*}$ & 0,13 \\
Benevolencia & $0,52^{*}$ & $-0,26$ \\
Universalismo & $0,57^{*}$ & $-0,35$ \\
Autodirección & $0,59 *$ & $-0,31$ \\
Estimulación & $0,47^{*}$ & $-0,11$ \\
Hedonismo & $0,42^{*}$ & 0,01 \\
Logro & 0,40 & $0,44 *$ \\
Poder & 0,09 & $0,78^{*}$ \\
Seguridad & $0,47 *$ & 0,21 \\
\hline \multicolumn{2}{|c|}{ Varianza Explicada $=94,21 \%$} \\
\multicolumn{2}{|c|}{ Wilk's Lambda $=0,13$} \\
\hline
\end{tabular}

Tabla 13. Análisis de la Eficiencia de la predicción del Análisis Discriminante.

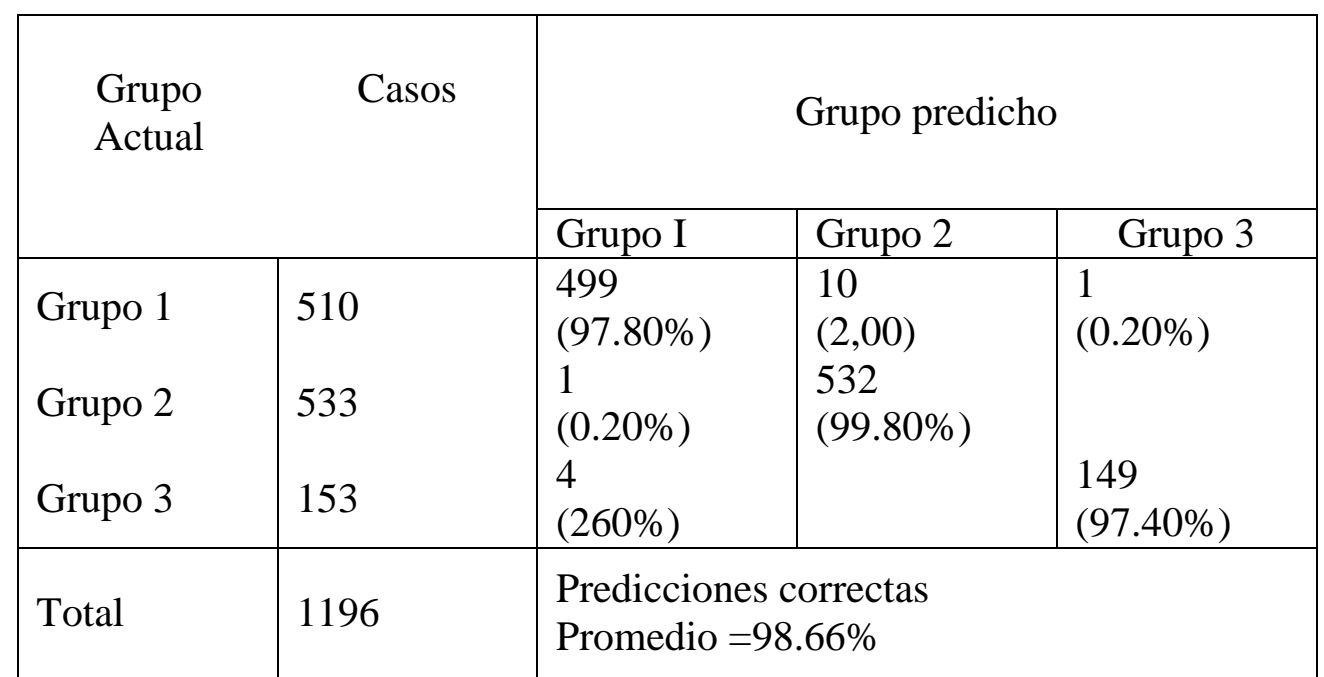




\section{DISCUSIÓN}

A través de este estudio, se pudo comprobar la estructura de valores en los estudiantes de secundaria de Lima Metropolitana, con base a una muestra representativa de alumnos.

Los resultados observados indicaron que los valores estudiados con el Cuestionario de Schwartz (PVQ), alcanzaron coeficientes de correlaciones Ítem-test que fueron significativas en todos los casos, así como elevados valores de con fiabilidad. Estos hallazgos estuvieron en concordancia con los resultados obtenidos en las investigaciones de Schwartz (1996), Schwartz y Sagie (2000), Schwartz, Sagiv \& Boehnke (2000) y Schwartz, Melech, Lehmann, Burgess, Harris, \& Owens (2001).

Del mismo modo el resultado del análisis estructural realizado a través del método del Análisis del Espacio Mínimo (SSA), permitió identificar una distribución de los valores similar a la que propuso la teoría de Schwartz, hecho que también ocurrió en los trabajos desarrollados por Schwartz y Sagie (2000), por Schwartz, Sagiv \& Boehnke (2000) y por Schwartz, Melech, Lehmann, Burgess, Harris, \& Owens (2001). Estos resultados permitieron demostrar la validez y generalidad de la concepción teórica de los valores desarrollada por Schwartz, así como la factibilidad de aplicarlo adecuadamente en nuestro contexto.

El análisis de los puntajes alcanzados por los valores permitió apreciar en general como se organizan, siendo los más valorados, la autodirección, el universalismo y la benevolencia, notándose que estos valores presentaron un claro carácter prosocial y que coinciden con los hallazgos reportados por Kornbilt (2000). En el otro extremo encontramos que los valores que fueron menos calificados por los estudiantes de Sto. de secundaria son los de conformidad, tradición y poder, lo que demostró ser coherente con los hallazgos alcanzados.

Cuando se analizaron los resultados de acuerdo a los tipos motivacionales que están formados con base a la estructura de valores, notamos que por encima de cualquier otro, apareció el valor de autodirección que conformó la dimensión de apertura al cambio y que se contrapone a la dimensión de conservación que incluye a los valores de conformidad y tradición. En tanto que los valores de universalismo y benevolencia, formaron la dimensión de autotranscendencia, y que se opuso a la dimensión de promoción personal que incluyó al valor de poder.

Los resultados del contraste de la primera hipótesis que aludió a la comparación entre estratos socioeconómicos, permitió apreciar la existencia de diferencias en algunos valores con predominancias diferenciadas entre ellos. Así se encontró que en el estrato socioeconómico bajo predominaron los valores de tradición y seguridad, es decir que en estos alumnos se presentó el tratar de respetar y comprometerse en aceptar las costumbres e ideas de la cultura tradicional, así como el lograr una mayor seguridad en los aspectos sociales e interpersonales, es por ello que podemos indicar que en ellos predominaron los valores asociados con la dimensión de conservación pero con un carácter colectivo.

Una situación totalmente distinta ocurrió en los estratos alto y medio, en quienes predominaron de manera conjunta la autodirección, el hedonismo y el logro, es decir que se dieron las motivaciones de las dimensiones de apertura al cambio y de promoción personal, es decir que expresaron un mayor interés por adaptarse a los cambios de la sociedad y lograr el desarrollo personal.

Otro dato relevante con relación al estrato socioeconómico radica en que también existieron valores predominantes en cada uno de ellos. Así en el estrato alto predominó, la benevolencia es decir los aspectos relacionados con preservar y reforzar el bienestar de las personas a quienes son considerados miembros de un mismo grupo social y que estuvo 
asociada a la dimensión a la autotrascendencia; en tanto que en el estrato medio predominó el valor de poder, es decir la búsqueda de lograr prestigio y posición social, lo cual supone una situación de búsqueda de ascenso social.

Es gracias a estos resultados que podemos considerar que la primera hipótesis es válida y que efectivamente la pertenencia a un determinado estrato socioeconómico pudo condicionar el desarrollo de ciertos valores, los cuales probablemente respondieron a las exigencias adaptativas del entorno social donde se desarrollan los alumnos.

Respecto a la segunda hipótesis, que indicó que existieron diferencias en los valores por sexo, los resultados indicaron que existieron diferencias significativas en el caso de benevolencia, seguridad, universalismo, logro y poder. El análisis pormenorizado del sentido de las diferencias que fueron observadas, permitió denotar que en las mujeres predominaron los valores de benevolencia, seguridad y universalismo, es decir que predominó el interés por preservar y reforzar el bienestar de las personas cercanas con quienes tiene un contacto personal frecuente, conseguir seguridad armonía y estabilidad en la sociedad y la comprensión, aprecio, tolerancia y atención hacia el bienestar de toda la gente y hacia la naturaleza, lo cual indica que tuvieron una mayor importancia las dimensiones de trascendencia y de conservación. Mientras que en los hombres predominaron los valores de logro y poder, lo que indicó la importancia de obtener éxito personal como resultado de la demostración de competencia según las normas sociales y la búsqueda de posición y prestigio social, demostrando que se presentó con mayor fuerza la dimensión de promoción personal. Los hallazgos encontrados indicaron que la segunda hipótesis también fue válida.

Los resultados observados son muy interesantes porque en primer lugar indicaron la existencia de diferencias por sexo, resultado poco frecuente en los estudios realizados previamente, como lo reportaron en sus estudios en estudiantes españoles Martínez Sánchez, Flores Nistal \& Pérez Acosta (1999) Y en estudiantes argentinos Kornbilt (2000), lo cual demostraría el impacto que produce el contexto sociocultural en que se desenvuelven los participantes, pues éstos resultados corresponderían a los principales procesos de diferenciación de los roles sexuales, pues en los contextos latinos se encuentran claramente diferenciados los patrones de comportamiento y acción por sexo tal como lo indican Matud, Rodriguez, Marrero \& Carballeira (2002).

La existencia de grupos característicos de acuerdo a su forma de responder a los valores, es otro hallazgo relevante en la medida que permite demostrar que la sociocultura ejerce un efecto diferenciador sobre los alumnos ya sea que pertenezcan a un determinado estrato socioeconómico o sean de un determinado sexo, lo cual vislumbra la existencia de nuevas interrogantes que trataremos de responder en futuras investigaciones.

\section{CONCLUSIONES}

Los resultados de la investigación indican que:

1. Los valores medidos por el Cuestionario de Valores de Schwartz (PVQ), presentan confiabilidad por consistencia interna.

2. El Cuestionario de Valores de Schwartz (PVQ), presenta validez de constructo.

3. La teoría de los valores de Schwartz es factible de ser aplicada en nuestro medio.

4. El análisis estructural revela que los valores se orientan de acuerdo a la teoría con excepción de benevolencia y universalismo

5. Los valores de los alumnos de quinto año presentan una distribución normal.

6. Los valores de tradición y seguridad predominan en los alumnos del estrato socioeconómico bajo. 
7. El valor de poder predomina en los alumnos del estrato socioeconómico medio.

8. El valor de benevolencia predomina en los alumnos del estrato socioeconómico alto.

9. Los valores de autodirección, hedonismo y logro predominan en los alumnos de estratos socioeconómicos altos y medio.

10. Los valores de benevolencia universalismo y seguridad predominan en las mujeres.

11. Los valores de logro y poder predominan en los varones.

12. Los valores más calificados por los estratos socioeconómicos medio y alto son autodirección, benevolencia y universalismo.

13. En la muestra estudiada existen tres tipos de grupos según la forma de responder a los valores

\section{REFERENCIAS BIBLIOGRAFICAS}

Alarcón, R. (1991) Métodos y diseños de investigación del comportamiento. Lima: Universidad Peruana Cayetano Heredia, Fondo Editorial.

Apoyo Opinión y Mercado S.A. (1999). Niveles socio económicos en Lima Metropolitana. Lima.

Bilsky, W. (2002) La Teoría de las Facetas: Informaciones Básicas y Aplicaciones Paradigmáticas. Münster: Berichte aus dem Psychologische Institut IV.

Bilsky, W. \& Koch, M. (2002). On the content and structure of values: Universals or methodological artifacts? In J. Blasius, J. Hox, E. deLeeuw \& P. Schmidt (Eds.), Social science methodology in the New MiJlennium. Updated and extended proceedings of the Fifth International Conference on Logic and Methodology, October 3-6, 2000, Cologne, Germany [CD-ROM]. Leverkusen: Leske.

Harnmond, S. M. (1999) The Psychometric Analysis Package (Pap-Facet) Version 2.1. University of Surrey

Hair, J. F., Anderson, R. E., Tatham, R. L. \& Black, W. C. (1999). Análisis Multivariante. México: Prentice Hall. Intemational.

Heady, R. B. \& Lucas, J. (2003). PERMAP 11.0 Operation Manual. Lousiana: Lafayette Press.

Kline, P. (1995). The Handbook of Psychological Testing. London: Routledge.

Kornbilt, A. L. (2000) Valores individuales y colectivos en una muestra de jóvenes argentinos. Instituto Gino Germani. Facultad de Ciencias Sociales. Universidad de Buenos Aires

Kluckohn, C (1951) Value and value orientations in the theory of action. En T Parsons \& Shills (eds). Towards a general theory of action. Cambrige. Harvard u.Press.

Martínez, R. (1996). Psicometría: Teoría de los Tests Psicológicos y Educativos. Madrid: Editorial Síntesis.

Martínez Sánchez, E.; Flores Nistal, M.A., Pérez Acosta, M.A. (1999). Sistema de Valores Personales en los jóvenes universitarios. Iberpsicología, Universidad Complutense (Madrid).

Matud, M. P., Rodríguez, c., Marrero, R. \& Carballeira, M. (2002). Psicología del Género: implicaciones en la vida cotidiana. Madrid: Editorial Biblioteca Nueva.

MDS(X) (2001) MINI-SSA (N): Michigan Israel Netherlands Integrated Smallest Space Analysis (Nijmegen) Users manual. Edimburgh: MDS(x) Press.

Muñiz, J. (1994). Teoría Clásica de los tests. Madrid: Pirámide.

Muñiz, J. (1996). Psicometría. Madrid: Uruversitas. Rokeach, M. (1973). The Nature of Human Values. New York: Free Press.

Sagiv, L. \& Schwartz, S. H. (1999) Value Priorities and Subjective Well-Being: Direct 
Relations and Congruity Effects. European- Joumal-of-Social- Psychology. Mar-Apr; Vol 30(2): 177-198.

Sánchez, C. H. Y Reyes, M. C. (1990). Metodología y Diseños en la Investigación Científica: Aplicadas a la Psicología, Educación y Ciencias Sociales. Lima: INIDE.

Schwarlz, SR \& Bilsky, W. (1987). Towards a slruclural model of human values. Journal of Personality and Social Psychology, 53, 550-562.

Schwarlz, S.H. \& Bilsky, W. (1990). Toward a theory of the universal content and structure of values : Extensions and crosscultural replications. Joumal ofPersonality and Social Psychology, 58,878-891.

Schwartz, S.H. (1992). Universals in the content and structure of values : Theoretical advances and empirical test in 20 countries. En M. Zanna (Ed.), Advances in Experimental Social Psychology : Vol. 25 (pp. 1-65). Orlando, FL : Academic Press.

Schwartz, S.H. (1994). Are there universal aspects in the structure and content of human values JournaJ of Social Issues, 50, 19-45.

Schwartz, S.H. (1996). Yalue priorities and behavior : Applying a Theory ofIntegrated Yalue Systems. En C. Seligman, J.M. Olson \& M.P. Zanna (Eds.), The Psychology of Yalues : The Ontario Symposium, Vol. 8 (pp. 1-24). Mahwah, NJ :Lawrence Erlbaum.

Schwartz, S. H., Sagiv, L. (1995) Identifying culture-specifics in the content and structure of values. Journal of Cross Cultural Psychology. Jan, Vol 26(1): 92116.

Schwartz S. H., Sagiv, L. \& Boehnke (2000) Worries and Yalues. Journal-ofPersonality. Apr; Vol 68(2): 309-346.

Schwartz, S. H., Sagie, G. (2000). Yalue consensus and importance: A crossnalional study. Joumal ofCross Cultural Psychology. Jul, Vol 31(4): 465-497.

Schwartz, S. H., Melech, G., Lehmann A., Burgess, S., Harris, M. \& Owens Y (2001). Extending the Cross-Cultural Yalidity of lhe Theory of Basic Human Yalues with A Differenl Method of Measurement. Journal ofCross-Cultural Psychology, 32, Seplember 2001, 519-542

Siegel, S. y Castellan N. (1995). Estadística No paramétrica: Aplicada a las ciencias de la conducta. México: Editorial Trillas.

Williams, R.M. Jr. (1979). Change and stability in values and value systems : A sociological perspective. En M. Rockeach (Ed.), Understanding human values (pp. 1546). New York: Press. 